\title{
A Smart High-Throughput Experiment Platform for Materials Corrosion Study
}

\author{
Peng Shi, Bin Li, Jindong Huo, and Lei Wen \\ National Center for Materials Service Safety, University of Science and Technology Beijing, Beijing, China \\ Correspondence should be addressed to Peng Shi; shipengustb@sina.com
}

Received 6 August 2016; Accepted 4 October 2016

Academic Editor: Wenbing Zhao

Copyright ( 2016 Peng Shi et al. This is an open access article distributed under the Creative Commons Attribution License, which permits unrestricted use, distribution, and reproduction in any medium, provided the original work is properly cited.

\begin{abstract}
Materials corrosion study is based on plenty of contrast experiments. Traditional corrosion experiments are time-consuming and require manual corrosion grade evaluating during the experiment. To improve the efficiency of experiment, a high-throughput experiment platform is designed and accomplished. The platform mainly consists of high-throughput corrosion reaction facility, data acquisition system, and data processing system. The corrosion reaction facility supports high-throughput materials corrosion reactions under various conditions. The data acquisition system is mainly responsible for capturing the images of samples' surface, collecting electrochemical signals, and storing them into the computer in real time. The data processing system treats the acquired data and evaluates the degree of materials corrosion in real time by program automatically. The platform not only reduces the occupation of the equipment but also improves the efficiency of sample preparation and experiment occurrence. The experimental data shows that the platform can accomplish high-throughput corrosion contrast experiment easily and reduce the time cost obviously.
\end{abstract}

\section{Introduction}

Corrosion is one of the most popular materials' failure causes. It will significantly reduce materials' strength, plastic, and toughness properties, shorten the service life, and cause catastrophic accidents [1]. To investigate the factors and mechanisms of materials corrosion, a lot of experiments must be conducted. A small change of the experimental parameters often means a series of new similar experiments, which will undoubtedly consume a large amount of resources. To improve the efficiency of this kind of experiments, highthroughput method is adopted.

The idea of high-throughput originates from combinatorial chemistry [2], aiming to shorten the product development period and reduce costs by large-scale chemical synthesis technology. Its essence is to accomplish large amount of repetitive experiments in parallel and obtain experimental results through the novel design of experiment scheme and equipment. High-throughput characterization technology has been widely used in gene sequencing, drug screening, and so on. In recent years, new materials development begins to adopt high-throughput technology to improve development efficiency [3]. However, there are few reports on high-throughput materials experiment method. In this paper, we try to improve the efficiency of materials corrosion experiment by high-throughput technology.

A high-throughput corrosion experiment platform has been designed and accomplished. The platform mainly comprises high-throughput corrosion reaction facility, data acquisition system, and data processing system. The corrosion reaction facility supports high-throughput materials corrosion reactions under various conditions. The data acquisition system is mainly responsible for capturing the images of samples' surface, collecting electrochemical signals, and storing them into the computer in real time. The data processing system treats the acquired data and evaluates the degree of materials corrosion in real time by program automatically.

The platform has accomplished some high-throughput materials corrosion experiments, which not only reduces the occupation of the equipment but also improves the efficiency of sample preparation and experiment conduction. Different 
from traditional artificial corrosion evaluation, the platform can treat the collected data and automatically generate corrosion evaluation results. The contribution of our method is that it provides the possibility of large-scale materials corrosion experiment and version-based data analysis.

The rest of the paper is arranged as follows. Section 2 introduces the related works about high-throughput experiment and corrosion image processing. Section 3 describes the structure and implementation of the platform. The key algorithm and programing method are presented in Section 4. The experimental procedure and result analysis are shown in Section 5. Section 6 analyzes the performance of our method. At last, Section 7 summarizes our contributions and points out the future work.

\section{Related Works}

2.1. High-Throughput Technology. High-throughput is a technology to execute parallel task with multicharacterization methods. It has been applied in several fields, including high-throughput sequencing, high-throughput screening, and combination technology. High-throughput sequencing [4] brings great promotion on the development of biological gene sequencing. It can measure millions of DNA molecules' sequence at the same time. This makes it possible to analyze the transcriptome and the genome of a species in detail. High-throughput screening [5] is a method for scientific experimentation especially used in drug discovery and relevant to the fields of biology and chemistry. It allows a researcher to quickly conduct millions of chemical, genetic, or pharmacological tests with robotics, data processing and control software, liquid handling devices, and sensitive detectors [6]. Combination technology, with multichannel parallel synthesis and high-throughput rapid characterization, can synthetize samples of different compositions fleetly by finite steps, investigate its structure and properties efficiently, and develop new materials with required properties finally [7].

2.2. Corrosion Image Processing and Evaluation. Image processing is a technology to analyze images and obtain some desired features by computer. It generally includes image compression, enhancement, restoration, matching, description, and recognition. Image description, matching, and recognition are usually adopted to accomplish some smart applications.

During the study of materials corrosion, the appearance of surface is important to evaluate the corrosion degree. The idea of image processing can be used in the grade evaluation of materials corrosion. In 1981, Itzhak et al. scanned the surface of AISI 304 material by digital scanner, which has been soaked for 20 minutes in $50^{\circ} \mathrm{C} 10 \% \mathrm{FeCl}_{3}$ solution [8]. The corrosive pitting is counted and the corrosion rate is calculated by computer program according to the scanned images. Codaroa et al. analyzed the appearance of pitting of Al-Ti alloy and gave a quantitative description method for pitting. The method can be utilized to represent the evaluation procedure of corrosion [9]. Wang et al. collected the morphology of sea water corrosion of carbon steel

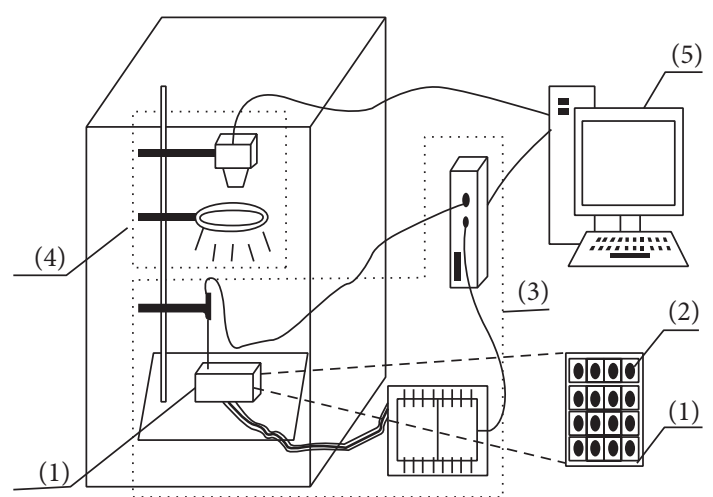

Figure 1: The structure of high-throughput corrosion experiment platform ((1) corrosion reaction facility, (2) specimen, (3) electrochemical signal acquisition system, (4) image acquisition system, and (5) computer).

[10]. They adopted grey correlation method to establish the relationship between the corrosion degree and the apparent color and edge. Xu et al. investigated the relationship between the apparent grey value of image and the depth of corrosive pitting in the specimen by fractal dimension method [11]. They found out that the relationship was nearly linear. In this paper, corrosion images are automatically processed in real time to evaluate the corrosion grade of specimens.

\section{Platform Structure}

With the idea of high-throughput, a smart materials' corrosion experiment platform is designed. It consists of highthroughput corrosion reaction facility, data acquisition system, and data processing system, shown in Figure 1. All the systems are connected to transfer signal and data.

3.1. High-Throughput Corrosion Reaction Facility. Highthroughput corrosion reaction facility indicates the container where the corrosion reaction occurs. We design two kinds of reaction units for high-throughput corrosion reaction: single-solution reaction unit and multiple-solution reaction unit.

3.1.1. Single-Solution Reaction Unit. The appearance of singlesolution reaction unit is shown in Figure 2. It uses corrosion resistant materials, such as epoxy resin, to package several specimens into one component. Single-solution reaction unit can only investigate the corrosion behaviors of different specimens in the same solution. The specimens in one component can be distinguished from materials, roughness, and other properties. Since the specimens are packaged, they can be treated together to improve the sample making efficiency, such as polishing and surface processing.

3.1.2. Multiple-Solution Reaction Unit. The appearance of multiple-solution reaction unit is shown in Figure 3. It is a reaction vessel consisting of a set of parallel tactic groove liquid pool arrays. Each liquid pool can contain different 


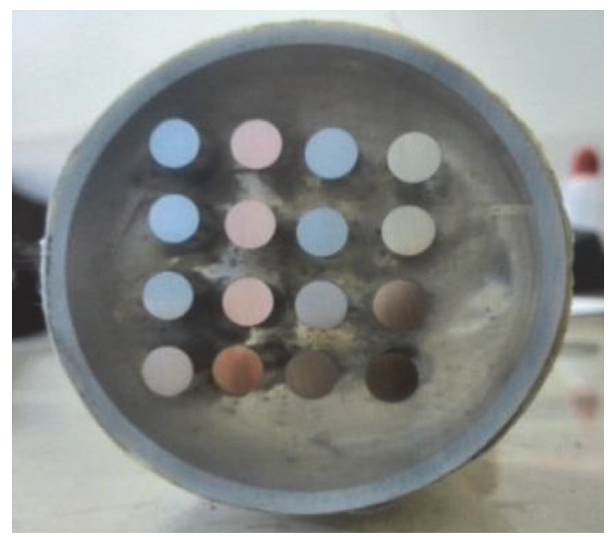

FIgURE 2: Single-solution reaction unit.
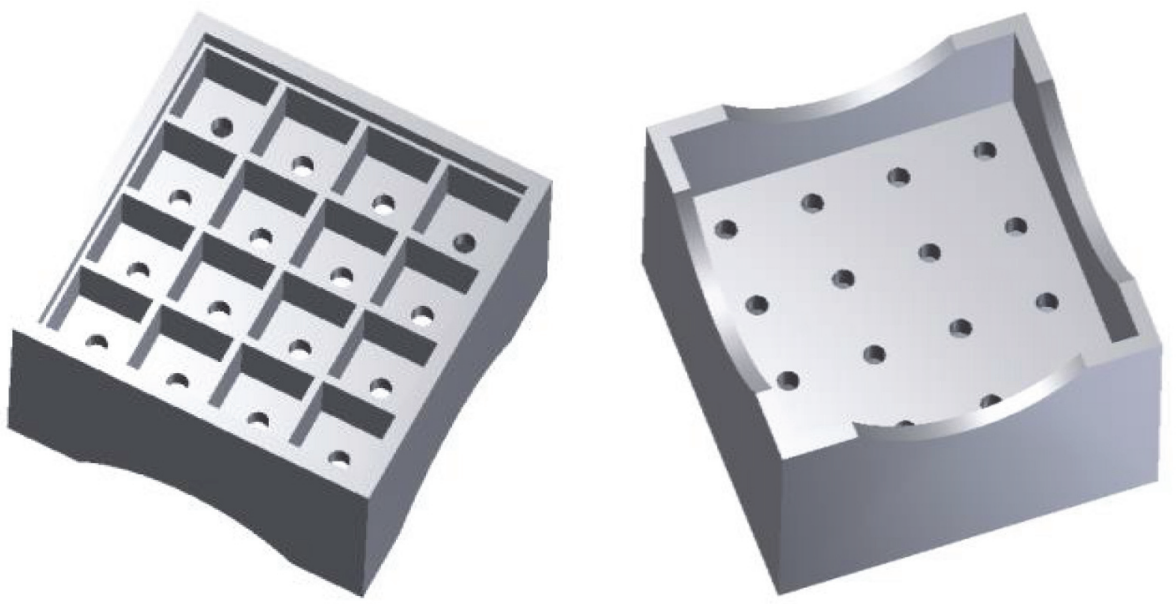

FIGURE 3: Multiple-solution reaction unit.

solutions during one experiment. Every specimen can be arranged into one liquid pool. After one specimen is put in, the bottom of the pool should be sealed up to avoid the leaking of solution. During the experiment procedure, each liquid pool can be plugged with an electrode. Thus, the electrochemical signals of every specimen can be collected independently. The advantage of multiple-solution reaction unit is that different specimens can be tested in different solutions at the same time. One experiment may produce independent image and electrochemical data of every specimen. It can obviously improve the corrosion reaction efficiency.

\subsection{Data Acquisition System}

3.2.1. Electrochemical Signal Acquisition System. Electrochemical signal is an important characterization parameter during materials corrosion reaction. It is usually detected by electrochemical workstation, representing the current and potential of the specimen and solution. The electrochemical signal acquisition system is composed of an electrochemical workstation, a reference electrode, and a multiplexer. The electrochemical workstation and reference electrode are working together to collect the signal of current and potential. In our high-throughput materials corrosion experiments, the two-electrode system was used to form a closed loop with a working electrode and a reference electrode. We use "Interface 5000" electrochemical workstation from Gamry ${ }^{\circledR}$ Company. The working electrode is a bolt type materials' electrode, and the reference electrode is commonly $\mathrm{Ag} / \mathrm{AgCl}$ electrode used in the study of electrochemical corrosion. $\mathrm{Ag} / \mathrm{AgCl}$ electrode can be utilized as a probe part of reference electrode in the solution.

Since high-throughput experiment needs to get the electrochemical signals of all the specimens, a multiplexer is designed to accomplish the function of parallel signal collection. The multiplexer is developed based on the circuit board. It consists of several input ports and one output port. Here we show a multiplexer with 16 input ports. All the specimens in the corrosion reaction unit can be connected with the input ports. The output port is connected with an electrochemical workstation to analyze the signal from the input ports. After circuit programing, we can realize the connecting between one input port and the output port at one moment. Thus, the multiplexers can take turns to query 16 input ports in accordance with the way specified by 


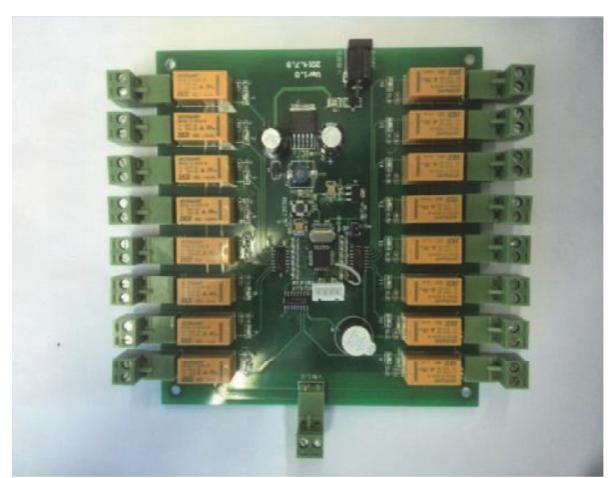

(a)

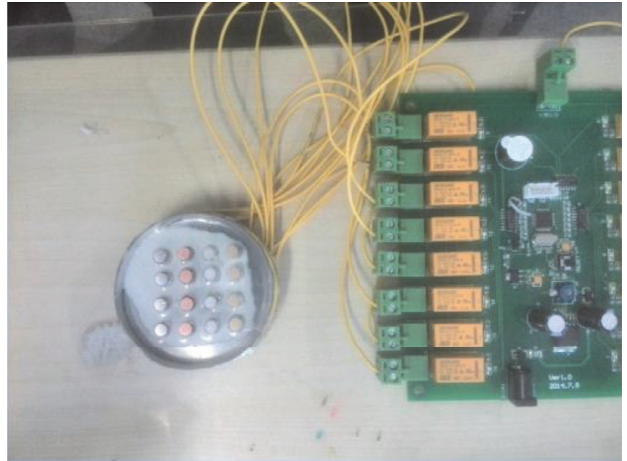

(b)

FIgURE 4: Multiplexer and its connection mode. (a) Multiplexer. (b) Connecting with specimens.

TABLE 1: Daheng camera parameters.

\begin{tabular}{lc}
\hline Name & Parameter \\
\hline Model & MER-500-7UC \\
Interface & Mini USB 2.0 \\
Resolution & $2592(\mathrm{H}) \times 1944(\mathrm{~V})$ \\
Frame rate & $7 \mathrm{fps}$ \\
Sensor & $1 / 2.5^{\prime \prime}$ CMOS \\
Pixel size & $2.2 \mu \mathrm{m} \times 2.2 \mu \mathrm{m}$ \\
Spectrum & Black-and-white $/$ color \\
\hline
\end{tabular}

the program. The appearance of the multiplexer is shown in Figure 4(a). Figure 4(b) shows the connecting mode with the experimental sample. With the help of the multiplexer, one single channel electrochemical workstation can accomplish the function of multichannel electrochemical workstation. Although there may have time delay on signal collection, it hardly influence the result because of slow corrosion action.

3.2.2. Image Acquisition System. Since the surface appearance of specimen is one important characterization for corrosion study, specimen surface image must be collected continuously. The image acquisition system is composed of a camera, a ring light, and a light shield.

We choose "MER-500-7UC" camera from Daheng ${ }^{\circledR}$ Graphics Company. The specific parameters of the camera are shown in Table 1 . The camera adopts USB 2.0 port to connect with computer and provides programing interface to control its action. Its size is only $29 \mathrm{~mm} * 29 \mathrm{~mm} * 29 \mathrm{~mm}$ and it is easy to be used in various environments.

For enough light on the specimens, we take LED ring light as a light source. It can supply an even light for the measured object. To avoid the influence from the environmental light, a light shielding cover was used. The stable light condition makes the direct comparison of images at different stages possible. Light shield selection was relatively simple, as long as it can cover the whole system and block the environmental light in.
3.3. Data Processing System. Data processing of our platform is automatically accomplished by programing, which is running on a computer. The data produced from the platform mainly includes the electrochemical signals and specimen surface images. The program can separate the electrochemical data of each specimen from the electrochemical workstation file, which contains the mixed data of all the specimens. The program can also process the surface image of specimens. The corrosion grade of each specimen is evaluated by image processing based program. The details of the method and algorithm will be introduced in Section 4 .

\section{Algorithm and Programing}

4.1. Electrochemical Signal Extracting. In our high-throughput experiment, the electrochemical data of all specimens are collected together. The electrochemical workstation collects signals sequentially and stores them into one DAT format file. To analyze the electrochemical signal of every specimen, the signal data of every specimen should be extracted from the DAT file. Based on the time stamp in the file and multiplexer, each specimen's electrochemical data can be attained by a program.

To avoid the error of data collecting, we set the data collecting with a higher frequency than the realistic demand. In the electrochemical data split program, the data with big error is picked out. An average data value of the remaining data with small error is adopted as the electrochemical signal value of the specimen by the program. After the program treatment, the data in DAT file is separated into several electrochemical signals. Every group of independent signals is corresponding to one specimen.

4.2. Image-Based Smart Corrosion Evaluation. The surface morphology and corrosion grade evaluation are important for the study of corrosion mechanism. During traditional corrosion experiment, the specimen corrosion grade is evaluated manually. With our smart high-throughput experiment platform, the specimen corrosion grade can be evaluated by the program automatically. 
In the past decades, image processing technology has been successfully applied in corrosion description and evaluation. Most of the related works focused on the analysis of grey image. Here we give a quick corrosion degree evaluation method for color corrosion image. For the RGB image, each pixel point has three color channels: red, green, and blue. Each color channel can take any value from 0 to 255 (with 8 bits' format), so the whole color space is a total of $256^{3}$ (about 16 million) kinds of color combination. The calculation of comparing the distribution of these 16 million combinations is too large, so we design a quick method to evaluate the color image by characteristic value. The RGB value from 0 to 255 is divided into four ranges: 0 to 63 is the first range; 64 to 127 is the second range; 128 to 191 is the third range; and 193 to 255 is the fourth range. Each color channel can be divided into four districts, so $4^{3}$ (64) kinds of color combinations can be reformed. The 64 kinds of combinations can be regarded as a space of 64 dimensions.

Here we define the concept of characteristic vector $C$ of an image. $C$ is a 64-dimension vector. The element of $C$ is the total number of pixels with the color of certain dimension, denoted as $c_{i}$. Thus a color image can be represented by a 64dimensional vector. For example, the characteristic vector of the left top brass specimen in Figure 8(a) can be denoted as

$$
C=(7414,230,0,0,8, \ldots, 109,0,0,3415,53929) \text {. }
$$

The details of RGB district and characteristic vector are shown in Table 2. The elements in column " $c$ " compose a vector, that is, the characteristic vector. It can be regarded as a "fingerprint" of an image. The similarity between two images can be determined by comparing their characteristic vectors [12]. In this way, we can improve the efficiency of image similarity determination while ensuring the relative uniqueness.

To quickly evaluate the corrosion grade of a specimen, we establish a standard corrosion image database. The database consists of a series of standard corrosion images with manual corrosion grade evaluation. The characteristic vectors of the images are also stored in the database. The main idea of quick corrosion grade evaluation is to specify one standard image's grade to an image, whose characteristic vector is the nearest to the standard image. The procedure of corrosion grade evaluation based on characteristic vector similarity mainly consists of the following five steps:

(1) Load the corrosion images to be evaluated.

(2) Calculate the characteristic vector of the image to be evaluated.

(3) Compare the calculated characteristic vector with that of the standard image one by one.

(4) Find out the standard image with the nearest characteristic vector.

(5) Output the corrosion grade of the standard image as that of the input image.

Based on the procedure above, we have developed a program to calculate corrosion grade of collected images. Consequently, the high-throughput experiment platform can output the corrosion grade of every specimen in real time.
TABLE 2: RGB and characteristic vector of image.

\begin{tabular}{|c|c|c|c|}
\hline $\mathrm{R}$ & $\mathrm{G}$ & B & $c$ \\
\hline 0 & 0 & 0 & 7414 \\
\hline 0 & 0 & 1 & 230 \\
\hline 0 & 0 & 2 & 0 \\
\hline 0 & 0 & 3 & 0 \\
\hline 0 & 1 & 0 & 8 \\
\hline 0 & 1 & 1 & 372 \\
\hline 0 & 1 & 2 & 88 \\
\hline 0 & 1 & 3 & 0 \\
\hline 0 & 2 & 0 & 0 \\
\hline 0 & 2 & 1 & 0 \\
\hline 0 & 2 & 2 & 10 \\
\hline 0 & 2 & 3 & 1 \\
\hline 0 & 3 & 0 & 0 \\
\hline 1 & 0 & 0 & 891 \\
\hline 1 & 0 & 1 & 13 \\
\hline 1 & 0 & 2 & 0 \\
\hline 1 & 0 & 3 & 0 \\
\hline 1 & 1 & 0 & 592 \\
\hline 1 & 1 & 1 & 3462 \\
\hline 1 & 1 & 2 & 355 \\
\hline 1 & 1 & 3 & 0 \\
\hline 1 & 2 & 0 & 0 \\
\hline 1 & 2 & 1 & 101 \\
\hline 1 & 2 & 2 & 882 \\
\hline 1 & 2 & 3 & 53110 \\
\hline 1 & 3 & 0 & 11053 \\
\hline 2 & 0 & 0 & 1146 \\
\hline 2 & 0 & 1 & 0 \\
\hline 2 & 0 & 2 & 0 \\
\hline 2 & 0 & 3 & 0 \\
\hline 2 & 1 & 0 & 2552 \\
\hline 2 & 1 & 1 & 9040 \\
\hline 2 & 1 & 2 & 47 \\
\hline 2 & 1 & 3 & 0 \\
\hline 2 & 2 & 0 & 0 \\
\hline 2 & 2 & 1 & 8808 \\
\hline 2 & 2 & 2 & 8 \\
\hline 2 & 2 & 3 & 0 \\
\hline 2 & 3 & 0 & 16 \\
\hline 3 & 0 & 0 & 11 \\
\hline 3 & 0 & 1 & 0 \\
\hline 3 & 0 & 2 & 0 \\
\hline 3 & 0 & 3 & 0 \\
\hline 3 & 1 & 0 & 856 \\
\hline 3 & 1 & 1 & 1376 \\
\hline 3 & 1 & 2 & 0 \\
\hline 3 & 1 & 3 & 0 \\
\hline 3 & 2 & 0 & 0 \\
\hline 3 & 2 & 1 & 3650 \\
\hline 3 & 2 & 2 & 6260 \\
\hline 3 & 2 & 3 & 109 \\
\hline 3 & 3 & 0 & 0 \\
\hline
\end{tabular}

\section{Experiments and Data Analysis}

With the smart high-throughput experiment platform, we have conducted several experiments of materials corrosion in $\mathrm{NaCl}$ solution. 


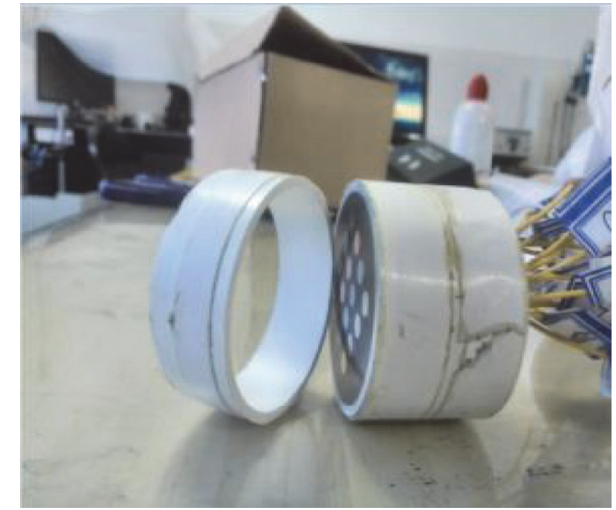

(a)

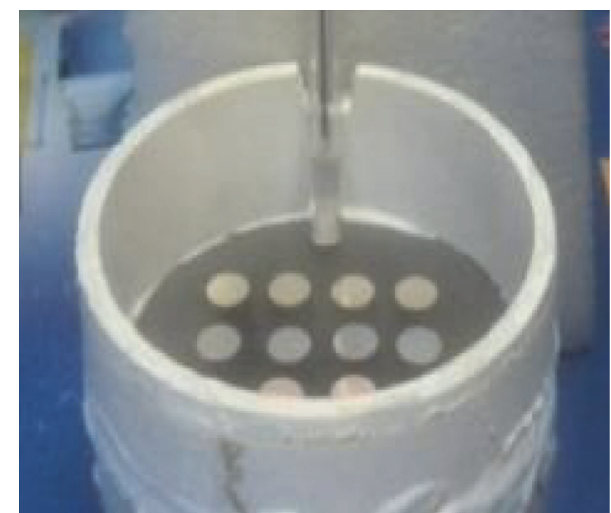

(b)

FIGURE 5: The solution container on single-solution corrosion unit. (a) Sticking method. (b) Solution container with an electron.

TABLE 3: The polishing sandpaper types and surface roughness.

\begin{tabular}{lcccc}
\hline Sandpaper type & $200 \#$ & $400 \#$ & $800 \#$ & $1500 \#$ \\
Surface roughness $/ \mu \mathrm{m}$ & 75.0 & 35.0 & 21.8 & 12.6 \\
\hline
\end{tabular}

5.1. Experiment Preparation. Four kinds of materials, including aluminum, brass, copper, and steel, are selected as the objects to be investigated. Every kind of material is made into four specimens as parallel reference separately. They are made into bar sample, with $5 \mathrm{~mm}$ diameter and $20 \mathrm{~mm}$ length. The intersecting surface is polished as the corrosion surface, by $200 \#, 400 \#, 800 \#$, and 1500\# sandpaper, respectively. Thus the effect of surface roughness on materials corrosion can be studied. The polishing sandpaper types and surface roughness are shown in Table 3.

The corrosion experiment is conducted in $3.5 \% \mathrm{NaCl}$ solution. Here we use the single-solution corrosion reaction unit as reaction container. After the specimens are polished, a pipe made of PTFE (Polytetrafluoroethylene) is stuck to the package surface by 704 silicone rubber. Then the $\mathrm{NaCl}$ solution can be contained in the pipe space. The appearance of stuck single-solution unit is shown in Figure 5(a). A reference electrode is plugged into the container to collect the electrochemical signals, shown in Figure 5(b).

\subsection{Experimental Data}

5.2.1. Electrochemical Signal. The experiment monitors the open circuit potential signal of the specimens by twoelectrode system. Ag/AgCl electrode is adopted as the reference electrode of the corrosion system. The reference electrode is connected with the electrochemical workstation. The specimens can be regarded as working electrode. All the working electrodes are connected with the input ports of the multiplexer one by one with extension wire. The output port of the multiplexer is directly connected with the Gamry electrochemical workstation. Thus the specimens, electrochemical workstation, multiplexer, and reference electrode compose a connected loop.

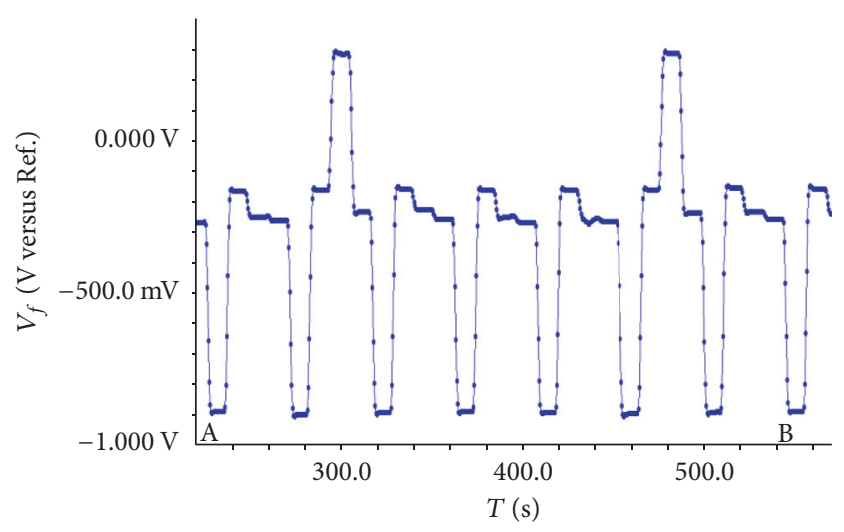

FIGURE 6: Open circuit potential of a full sampling period.

In order to obtain stable data output, we maintain 5second period of switching on with each specimen by multiplexer control program. At the same time, the Gamry potential signal scanning period is set to 0.5 seconds. Consequently, the signal scanning may complete about 10 times in each specimen's switching on interval. After removing the data with big offset, the average of the remaining data is calculated. The average value is specified as the potential value of the specimen in the switching on period. The platform can accomplish a complete scan circle for all the specimens in 80 seconds. The data of one complete circle is shown in Figure 6. Every point in the diagram is original data from the electrochemical workstation.

5.2.2. Surface Image. During the experiment, the image acquisition system records the specimens' surface image automatically. Since the corrosion action is not fast, the system captures the surface image once per hour by the program. The corrosion morphology images of the specimens within $0 \sim 7$ hours are shown in Figure 7. From the images, researchers can investigate the corrosion morphology. The corrosion morphology comparison on different materials and different corrosion time can be executed easily. 


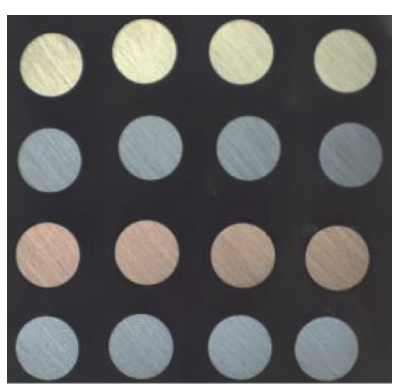

(a)

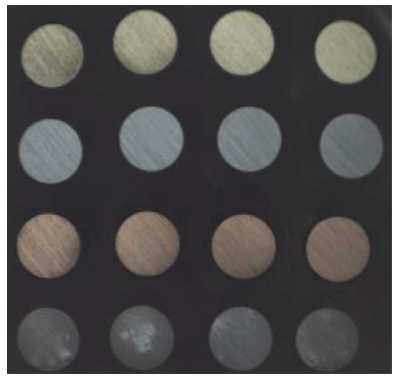

(e)

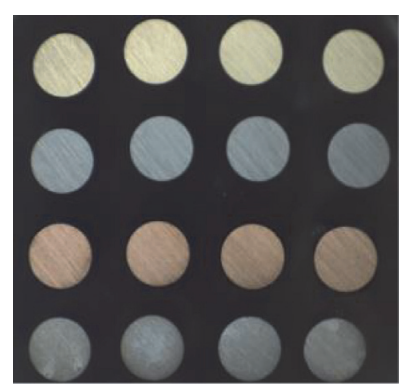

(b)

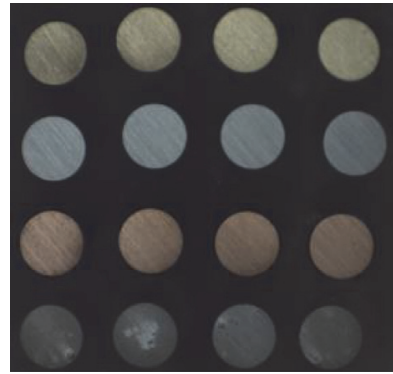

(f)

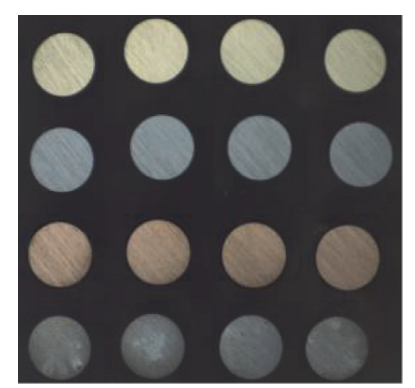

(c)

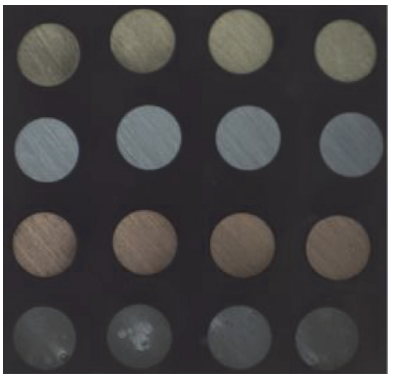

(g)

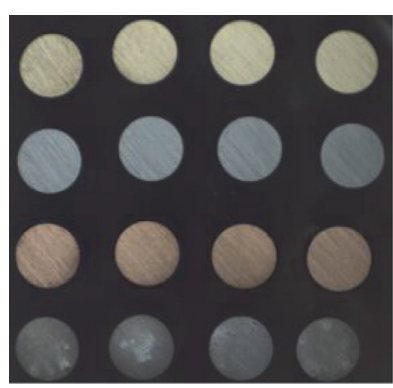

(d)

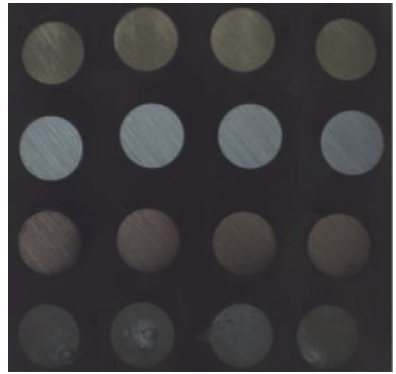

(h)

FiguRE 7: High-throughput corrosion images of one-hour interval. (a) $t=0 \mathrm{~h}$, (b) $t=1 \mathrm{~h}$, (c) $t=2 \mathrm{~h}$, (d) $t=3 \mathrm{~h}$, (e) $t=4 \mathrm{~h}$, (f) $t=5 \mathrm{~h}$, (g) $t=6 \mathrm{~h}$, and $(\mathrm{h}) t=7 \mathrm{~h}$.

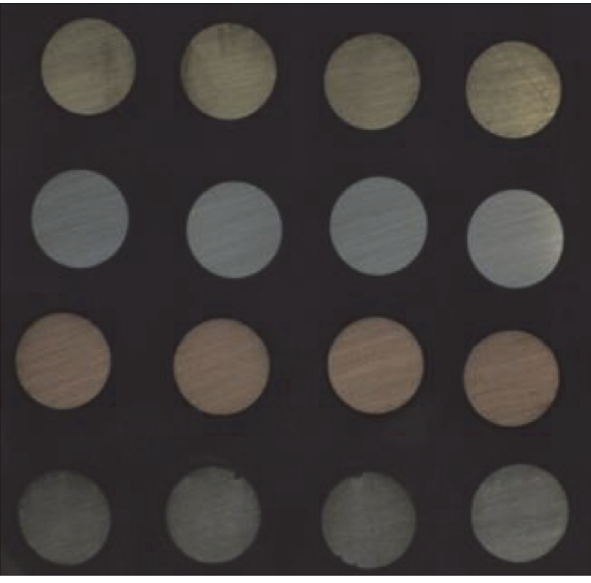

(a)
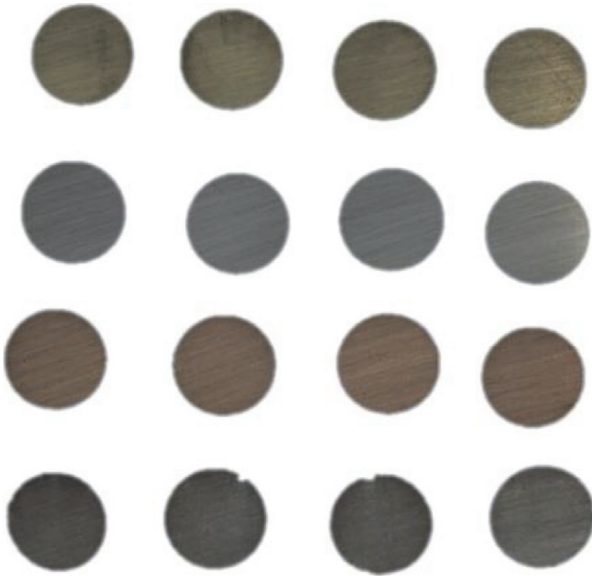

(b)

FIGURE 8: High-throughput image segmentation. (a) Image before segmentation. (b) Single specimen image.

5.3. Data Processing and Analysis. The data produced by high-throughput experiment is automatically processed in real time. Electrochemical signal processing is to separate the original data into 16 independent signals of every specimen. Image processing is mainly to evaluate the corrosion grade of every specimen.

5.3.1. Electrochemical Signal Processing. The electrochemical data of all the specimens collected by the electrochemical workstation are stored in a DAT format file. According to the program setting, the workstation collects data twice in one second, shown in Figure 6. The potential data of all the specimens is in one file. The data of each specimen is separated into several electrochemical signals by the program. The program considers two factors. One is the collecting time, by which the certain specimen can be located. The other is the data value. Because of the error of detecting, there may be some data with big offset. The program removes the offset data and calculates the average of the remaining normal data. The average value is specified as the potential value of the specimen in the switching on period. Thus every specimen contains a series of independent potential data.

The open circuit potential data after processing is shown in Figure 9. It presents the electrochemical signals of highthroughput experiment of carbon steel, brass, copper, and 


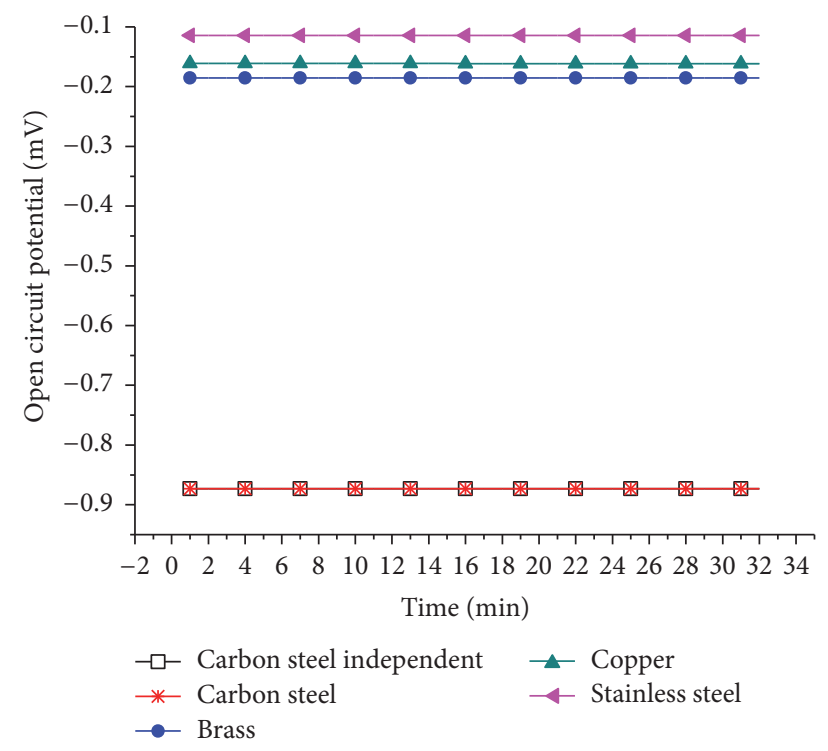

FIGURE 9: Electrochemical signals after processing.

stainless steel. To verify the correctness of high-throughput experiment, we conduct a carbon steel corrosion experiment with traditional independent experiment. The data of the independent experiment is also shown by black squares in Figure 9. From the comparison result, the independent experimental potential data is consistent with the data obtained by high-throughput experiment of the same materials in the same condition. Therefore, the phenomenon shows the correctness of high-throughput experiment in one respect.

5.3.2. Image Processing and Corrosion Grade. Since highthroughput experiment produces images with multiple specimens, the image of every single specimen should be extracted. Based on image edge detection method, the image of single specimen can be easily attained. The high-throughput image segmentation is shown in Figure 8. After the treatment by the program, the image of every specimen is extracted, shown in Figure 8(b).

Based on the standard GB/T 6461-2002, China standard of corrosion evaluation, the corrosion grade can be evaluated. The standard proposes two basic parameters: corrosion area ratio and corrosion grade, to evaluate the corrosion grade. The corresponding relationship between corrosion grade and corrosion area ratio is shown in Table 4. Corrosion area ratio is the percentage ratio between corrosion surface area and total surface area. The corrosion grade is from 1 to 10 . The higher the grade is, the more serious the corrosion status is.

To verify the correctness of corrosion evaluation, the corrosion images are also submitted to an expert of materials corrosion. The evaluation result of $7 \mathrm{~h}$ experiment by our program and the expert is shown in Table 5. From the table, it can be seen that there are some differences of the evaluated grade. It is because the expert uses his/her eyes to evaluate the corrosion grade by his/her experiences. After the confirmation of the expert, the program produced result is better than manual result.
TABLE 4: The corresponding relationship of grade value and corrosion area ratio.

\begin{tabular}{lc}
\hline Corrosion area ratio/\% & Corrosion grade value \\
\hline No corrosion & 10 \\
$0<A \leq 0.1$ & 9 \\
$0.1<A \leq 0.25$ & 8 \\
$0.25<A \leq 0.5$ & 7 \\
$0.5<A \leq 1.0$ & 6 \\
$1.0<A \leq 2.5$ & 5 \\
$2.5<A \leq 5.0$ & 4 \\
$5.0<A \leq 25$ & 3 \\
$25<A \leq 50$ & 2 \\
$50<A$ & 1 \\
\hline
\end{tabular}

TABLE 5: Corrosion evaluation result of $7 \mathrm{~h}$ experiment.

\begin{tabular}{lcc}
\hline $\begin{array}{l}\text { Specimen position in } \\
\text { the sample }\end{array}$ & $\begin{array}{c}\text { Grade value by } \\
\text { program }\end{array}$ & $\begin{array}{c}\text { Grade value by } \\
\text { expert }\end{array}$ \\
\hline 1,1 & 7 & 7 \\
1,2 & 7 & 7 \\
1,3 & 7 & 7 \\
1,4 & 6 & 7 \\
2,1 & 6 & 6 \\
2,2 & 6 & 6 \\
2,3 & 6 & 6 \\
2,4 & 5 & 6 \\
3,1 & 8 & 8 \\
3,2 & 7 & 8 \\
3,3 & 8 & 8 \\
3,4 & 8 & 8 \\
4,1 & 2 & 2 \\
4,2 & 3 & 3 \\
4,3 & 2 & 2 \\
4,4 & 4 & 4 \\
\hline
\end{tabular}

TABLE 6: Time cost comparison of one-by-one, parallel and highthroughput experiment mode.

\begin{tabular}{lccc}
\hline Steps & $\begin{array}{c}\text { One-by- } \\
\text { one/minute }\end{array}$ & Parallel/minute & $\begin{array}{c}\text { High- } \\
\text { throughput/minute }\end{array}$ \\
\hline Polish & 960 & 960 & 240 \\
Encapsulation & 0 & 0 & 30 \\
Experiment & 6720 & 420 & 420 \\
Handle & 140 & 140 & 140 \\
\hline Total & 7820 & 1520 & 830 \\
\hline
\end{tabular}

\section{Performance Analysis and Discussion}

Compared with traditional corrosion experiments, the highthroughput experiment platform shows high efficiency, especially in time cost. Table 6 shows the time and equipment cost comparison of a typical high-throughput corrosion experiment with parallel experiment and one-by-one experiment. 
Here, the high-throughput experiment with 16 specimens is taken as an example. From the data in Table 6, it is clear that the high-throughput experiment costs much less time than traditional experiment way. The parallel experiment and high-throughput experiment cost less time than one-by-one experiment. However, more testing equipment is occupied by parallel experiment than one-by-one experiment and high-throughput experiment. Although the high-throughput method takes some time for sample packaging, it reduces the sample polishing time. The parallel experiment costs more time for polishing because the high-throughput sample can be polished together. From the total time cost data in Table 6, it is obvious that high-throughput experiment has the highest efficiency. From the analysis above, it can be concluded that the high-throughput experiment method designed in this paper has a great advantage in the equipment occupation and the overall time consumption.

\section{Conclusion and Future Work}

In this paper, we propose a smart high-throughput experiment platform for the research of materials corrosion. Intelligent analysis of the corrosion of materials was successfully accomplished by the experimental platform. The corrosion grade can be calculated by the program automatically. The calculated results are matched closely with the manual analyzed results. Unfortunately, for the experiment with little surface changing, the image-based corrosion evaluation may not work well.

In the future, we will try to collect other types of data during materials corrosion, such as microstructure, solution changes, and other characterization data. By analyzing the new types of data, it is expected to accomplish more comprehensive understanding of the mechanisms of materials corrosion. At the same time, through adding the new type of data to the standard library, the corrosion evaluation results can be more accurate.

\section{Competing Interests}

The authors declare that there is no conflict of interests regarding the publication of this paper.

\section{Acknowledgments}

This work is supported by "The Fundamental Research Funds for the Central Universities (FRF-TP-14-060A2)" of China-"The Research on Parallel Data Processing Method for Materials High-Throughput Experiment."

\section{References}

[1] N. Canter, "Materials corrosion preventives: protect materials and specific applications," Tribology \& Lubrication Technology, vol. 718, pp. 5-6, 2015.

[2] P. Dydio, P.-A. R. Breuil, and J. N. H. Reek, "Dynamic combinatorial chemistry in chemical catalysis," Israel Journal of Chemistry, vol. 53, no. 1-2, pp. 61-74, 2013.
[3] P. de Lima-Neto, A. N. Correia, R. A. C. Santana et al., "Morphological, structural, microhardness and electrochemical characterisations of electrodeposited $\mathrm{Cr}$ and Ni-W coatings," Electrochimica Acta, vol. 55, no. 6, pp. 2078-2086, 2010.

[4] L. Chen, L.-Y. Wang, S.-J. Liu et al., "Profiling of microbial community during in situ remediation of volatile sulfide compounds in river sediment with nitrate by high-throughput sequencing," International Biodeterioration \& Biodegradation, vol. 85, pp. 429-437, 2013.

[5] L. M. Junker and J. Clardy, "High-throughput screens for small-molecule inhibitors of Pseudomonas aeruginosa biofilm development," Antimicrobial Agents and Chemotherapy, vol. 51, no. 10, pp. 3582-3590, 2007.

[6] G. R. Eldridge, H. C. Vervoort, C. M. Lee et al., "Highthroughput method for the production and analysis of large natural product libraries for drug discovery," Analytical Chemistry, vol. 74, no. 16, pp. 3963-3971, 2002.

[7] P. A. White, A. E. Hughes, S. A. Furman et al., "High-throughput channel arrays for inhibitor testing: proof of concept for AA2024-T3," Corrosion Science, vol. 51, no. 10, pp. 2279-2290, 2009.

[8] D. Itzhak, I. Dinstein, and T. Zilberberg, "Pitting corrosion evaluation by computer image processing," Corrosion Science, vol. 21, no. 1, pp. 17-22, 1981.

[9] E. N. Codaroa, R. Z. Nakazatoa, A. L. Horovistizb, L. M. F. Ribeirob, R. B. Ribeirob, and L. R. O. Heinb, "An image processing method for morphology characterization and pitting corrosion evaluation," Materials Science and Engineering A, vol. 334, no. 1-2, pp. 298-306, 2002.

[10] S. Wang, D. Kong, and S. Song, "Diagnosing corrosion modality system of metallic material in seawater based on fuzzy pattern recognition," Acta Metallrugica Sinica, vol. 37, no. 5, pp. 517-521, 2001.

[11] S. Xu, Y. Weng, and X. Li, "Characterization for corrosion pit distribution by using fractal dimension of image," Journal of the Chinese Society of Corrosion and Protection, vol. 27, no. 2, pp. 109-113, 2007.

[12] K. Belaid, O. Echi, and R. Gargouri, "Two classes of locally compact sober spaces," International Journal of Mathematics and Mathematical Sciences, vol. 2005, no. 15, pp. 2421-2427, 2005. 

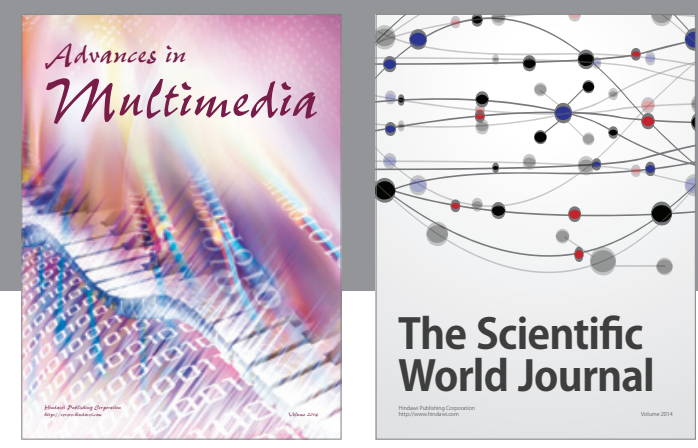

The Scientific World Journal
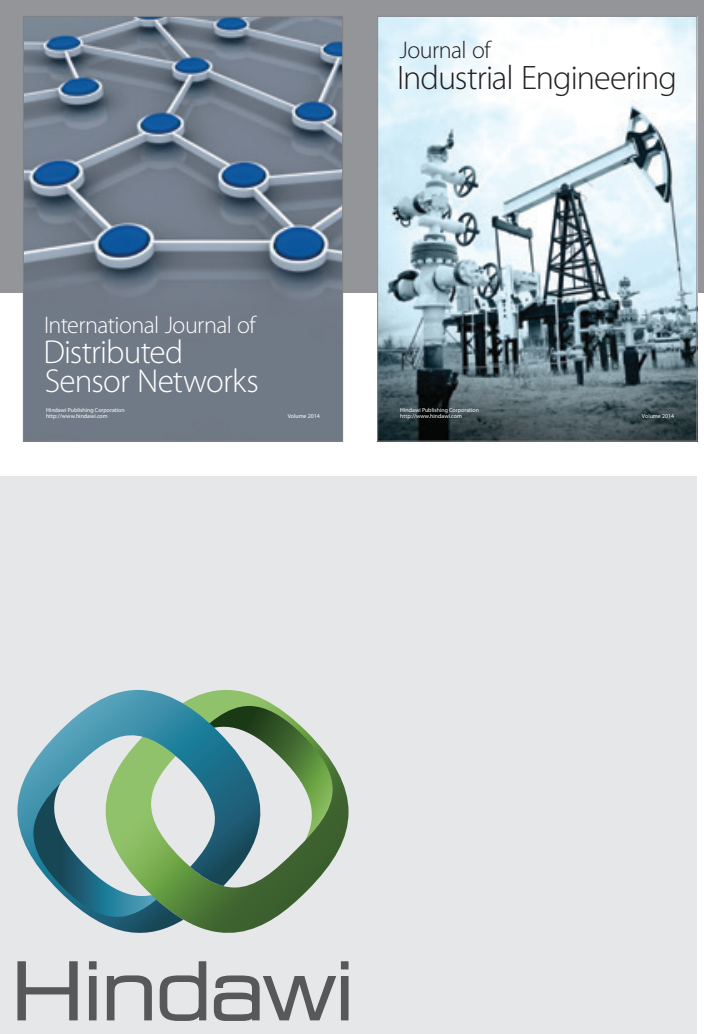

Submit your manuscripts at

http://www.hindawi.com

\section{Computer Networks} and Communications
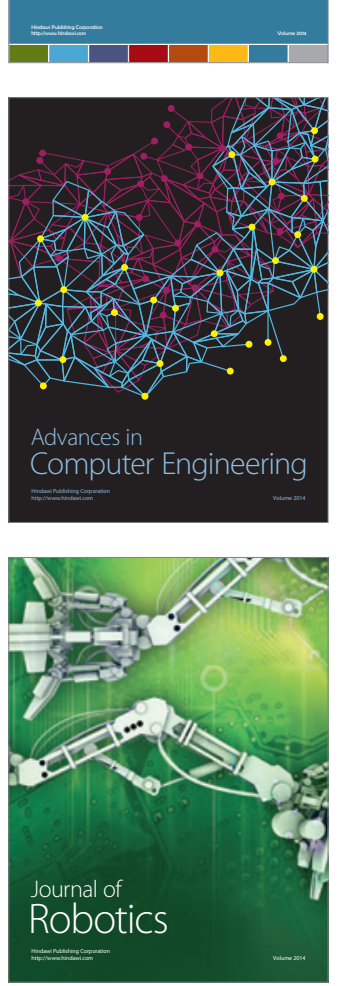
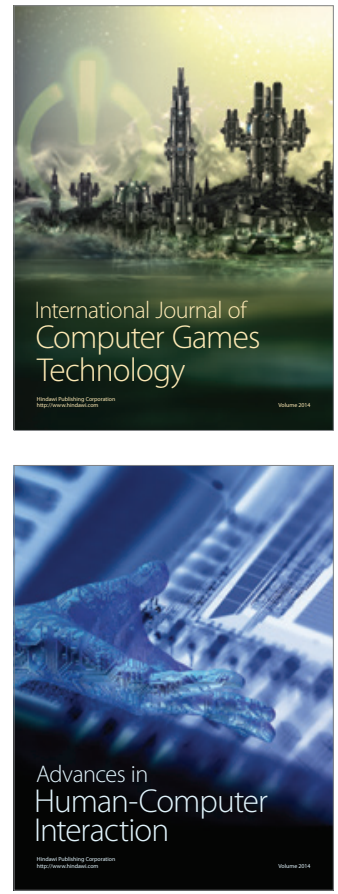
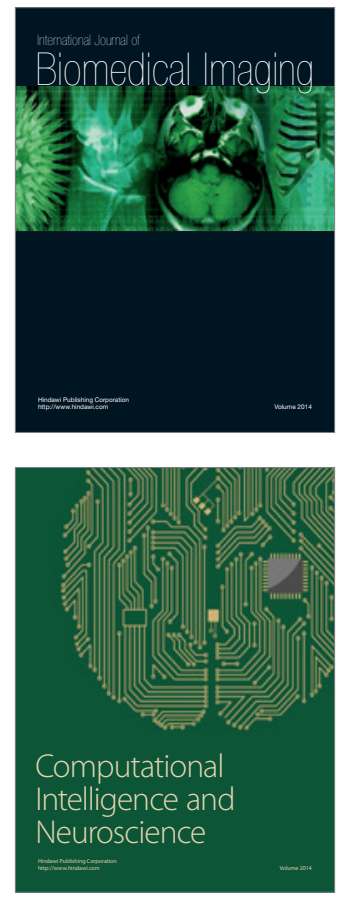
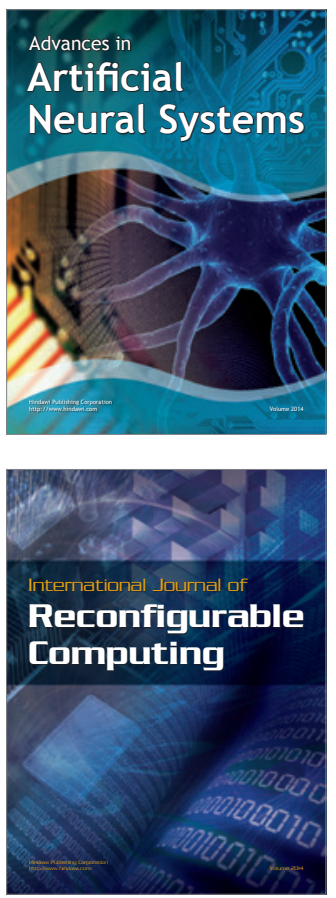
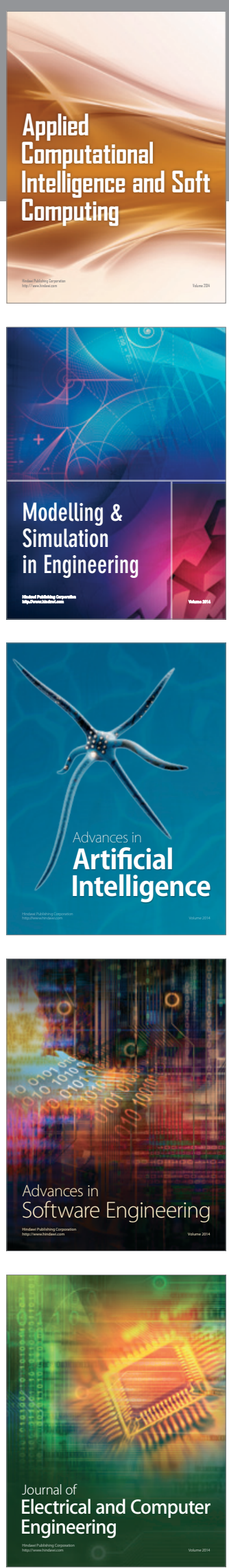